\title{
Structures, Energetics, and Dynamics of Helium Adsorbed on Isolated Fullerene Ions
}

\author{
Christian Leidlmair, ${ }^{1}$ Yang Wang, ${ }^{2}$ Peter Bartl, ${ }^{1}$ Harald Schöbel, ${ }^{1}$ Stephan Denifl, ${ }^{1}$ Michael Probst, ${ }^{1}$ Manuel Alcamí, ${ }^{2}$ \\ Fernando Martín, ${ }^{2,3}$ Henning Zettergren, ${ }^{4}$ Klavs Hansen, ${ }^{5}$ Olof Echt, ${ }^{1,6, *}$ and Paul Scheier ${ }^{1}$ \\ ${ }^{1}$ Institut für Ionenphysik und Angewandte Physik, Universität Innsbruck, Innsbruck, Austria \\ ${ }^{2}$ Departamento de Química C-13, Universidad Autónoma de Madrid, Madrid, Spain \\ ${ }^{3}$ Instituto Madrileño de Estudios Avanzados en Nanociencia (IMDEA-Nanociencia), Madrid, Spain \\ ${ }^{4}$ Department of Physics, Stockholm University, Stockholm, Sweden \\ ${ }^{5}$ Department of Physics, University of Gothenburg, Gothenburg, Sweden \\ ${ }^{6}$ Department of Physics, University of New Hampshire, Durham, New Hampshire, USA
}

(Received 23 September 2011; published 13 February 2012)

\begin{abstract}
Helium adsorbed on $\mathrm{C}_{60}{ }^{+}$and $\mathrm{C}_{70}{ }^{+}$exhibits phenomena akin to helium on graphite. Mass spectra suggest that commensurate layers form when all carbon hexagons and pentagons are occupied by one $\mathrm{He}$ each, but that the solvation shell does not close until $60 \mathrm{He}$ atoms are adsorbed on $\mathrm{C}_{60}{ }^{+}$, or 62 on $\mathrm{C}_{70}{ }^{+}$. Molecular dynamics simulations of $\mathrm{C}_{60} \mathrm{He}_{n}{ }^{+}$at $4 \mathrm{~K}$ show that the commensurate phase is solid. Helium added to $\mathrm{C}_{60} \mathrm{He}_{32}{ }^{+}$will displace some atoms from pentagonal sites, leading to coexistence of a registered layer of immobile atoms interlaced with a nonregistered layer of mobile atoms.
\end{abstract}

DOI: 10.1103/PhysRevLett.108.076101

Physisorption of helium and other inert gases on carbon materials gives rise to a wealth of intriguing phenomena. The binding of inert gases to graphite is relatively strong; for helium it exceeds the binding between the gas atoms [1]. In addition to coverage and temperature, the corrugation of the surface, its curvature, and dimensionality control the physical properties of the adsorbate. The beauty of carbon materials is the possibility to vary corrugation, curvature, and dimensionality over a wide range.

At one end of the spectrum lies the two-dimensional graphitic surface whose rather weak corrugation gives rise to the well-known $\sqrt{3} \times \sqrt{3}$ commensurate phase where one-third of the energetically preferred adsorption sites (at hexagon centers of the honeycomb lattice) is occupied [2]. The distance between adatoms in this phase is $4.26 \AA$ (3 times the carbon-carbon bond length); the coverage is 0.0637 atoms $/ \AA^{2}$. However, the first adsorbate layer is not complete until the coverage reaches 0.120 atoms $/ \AA^{2}$; other commensurate structures may form in the first and possibly in the second layer [2-4]. A single graphene sheet presents an even weaker corrugation than graphite; thus the $\sqrt{3} \times \sqrt{3}$ solid phase may compete with a two-dimensional superfluid phase [4].

Planar surfaces with strong corrugation are realized by ordered arrays of fullerenes (fullerite surfaces). A closepacked layer of $\mathrm{C}_{60}$ offers deep potential minima that form a honeycomb lattice with a spacing of $5.8 \AA$; Monte Carlo simulations predict an ordered phase of helium at low temperature [5].

The outer surface of a bundle of nanotubes provides a strongly corrugated, one-dimensional substrate to which helium binds more strongly than to graphite. Neutron diffraction studies show that at low coverage helium will form an array of one-dimensional liquids in the grooves
PACS numbers: 68.43.Bc, 36.40.Mr, 36.40.Qv, 61.48.-c

between tubes; with increasing coverage additional parallel lines of liquid helium will form in the grooves before a two-dimensional layer forms [6]. Details depend on the radius and chirality of the nanotubes $[7,8]$. An interesting feature common to nanotubes and fullerite surfaces is the existence of two kinds of corrugation, a strong one caused by the curved surfaces plus a weak one due to the hexagonal and, for fullerenes, pentagonal facets of the carbon network. The interplay between these two corrugations leads to interesting phenomena in theoretical studies $[5,8]$, but they are difficult to observe experimentally because of the unavoidable distribution of tube diameters and rotational orientation [9].

At the other end of the spectrum that started with graphitic surfaces are helium layers physisorbed on isolated nanotubes [10], planar aromatic molecules [11], and fullerenes $[12,13]$. The dimensionality of their surfaces changes from $0 \mathrm{D}$ or $1 \mathrm{D}$ to $2 \mathrm{D}$ as the size of the system increases. The resonance frequency of a single-walled suspended nanotube has been measured to monitor phase transitions in an adsorbate of argon and xenon [10]; the coupling of helium to gas-phase anthracene, phtalocyanine, and other planar aromatic molecules has been probed by high-resolution spectroscopy (see references in [11]).

In contrast, no experiments on helium attached to the outer surface of free fullerenes has yet been reported. $\mathrm{C}_{60}$ is easily embedded in helium nanodroplets, but mass spectrometric identification requires ionization which tends to boil off all helium atoms. Theoretical work that treats the fullerene surface as a continuum cannot explore structural order [12]. To the best of our knowledge only one theoretical study, of helium $+\mathrm{C}_{20}$ (which contains 12 pentagonal but no hexagonal facets), has explored the effect of corrugation on the adsorbate [14]. 
We present a combined experimental and theoretical study of helium bound to isolated $\mathrm{C}_{60}$ and $\mathrm{C}_{70}$ cations. Abrupt drops in the ion abundance beyond $\mathrm{C}_{60} \mathrm{He}_{32}{ }^{+}$and $\mathrm{C}_{70} \mathrm{He}_{37}{ }^{+}$signal a commensurate phase when each facet of carbon atoms is occupied by one helium atom. The structure may be viewed as the analogue of a hypothetical $1 \times 1$ phase which does not form on graphite because adjacent helium atoms would repel each other. Molecular dynamics (MD) simulations of $\mathrm{C}_{60} \mathrm{He}_{n}{ }^{+}$at $4 \mathrm{~K}$ reveal interesting properties of the adsorbate. The dissociation energy of $\mathrm{C}_{60} \mathrm{He}_{33}{ }^{+}$(for evaporation of one $\mathrm{He}$ ) is much smaller than that of $\mathrm{C}_{60} \mathrm{He}_{32}{ }^{+}$. Helium atoms added beyond $n=32$ do not yet build a distinct second layer; rather they displace atoms from pentagon sites which exhibit fluidlike behavior while atoms located at hexagons remain immobile.

Helium nanodroplets are produced by expanding helium from a stagnation pressure of $2 \mathrm{MPa}$ through a $5 \mu \mathrm{m}$ nozzle, cooled to about $8 \mathrm{~K}$, into vacuum. The average droplet size is $\approx 10^{6} \mathrm{He}$; droplets are superfluid with a temperature of $0.37 \mathrm{~K}$ [15]. The skimmed beam traverses a pickup region into which a small amount of $\mathrm{C}_{60}$ (MER, purity $99.9 \%$ ) or $\mathrm{C}_{70}$ (SES, 99\%) is vaporized. Doped helium droplets are ionized by electron impact at $70 \mathrm{eV}$ resulting in strong fragmentation (loss of most of the helium but no fragmentation of the fullerene). Cations are accelerated into the extraction region of a time-of-flight mass spectrometer equipped with a reflectron (Tofwerk AG, model HTOF). Details are described elsewhere [16].

A mass spectrum is displayed in Fig. 1. The dominant ion series is due to $\mathrm{C}_{60} \mathrm{He}_{n}{ }^{+} . \mathrm{C}_{60}{ }^{+}$at $720 \mathrm{u}$ (slightly higher for $\mathrm{C}_{60}{ }^{+}$containing one or more ${ }^{13} \mathrm{C}$ which has a natural abundance of $1.07 \%$ ) is off scale. The group of peaks around 738 is due to a $\mathrm{C}_{60}$-water complex. A weaker series, prominent below $720 \mathrm{u}$, arises from ${ }^{4} \mathrm{He}_{x}{ }^{+}$.

The region around $850 \mathrm{u}$ is reproduced in the upper inset. The first and most abundant peak in each group of mass peaks, connected by solid lines to the corresponding peaks in adjacent groups, represents ${ }^{12} \mathrm{C}_{60} \mathrm{He}_{n}{ }^{+}$. The

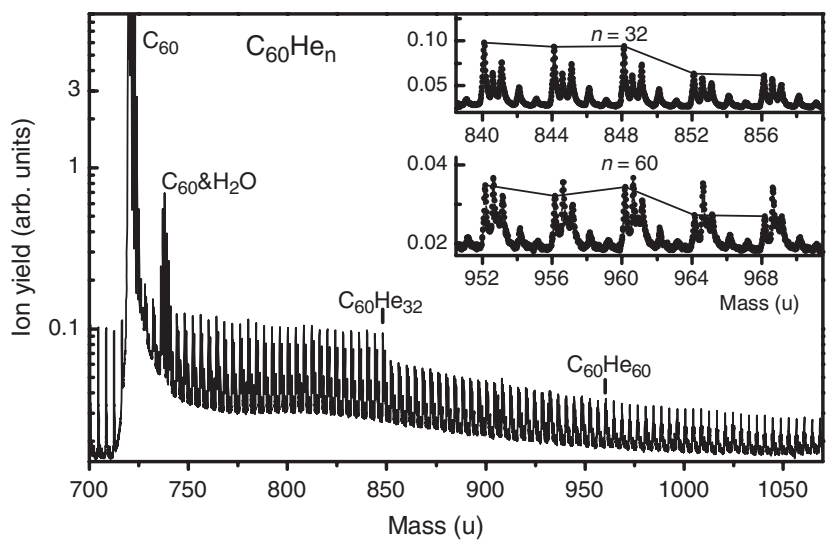

FIG. 1. Mass spectrum of helium droplets doped with $\mathrm{C}_{60}$. second peak in each group is $\mathrm{He}_{x}{ }^{+}$; it is shifted relative to ${ }^{12} \mathrm{C}_{60} \mathrm{He}_{n}{ }^{+}$by $0.469 \mathrm{u}$ (the mass difference between ${ }^{4} \mathrm{He}_{180}$ and $\left.{ }^{12} \mathrm{C}_{60}\right)$. The following peaks in each group arise from isotopologues of ${ }^{12} \mathrm{C}_{60} \mathrm{He}_{n}{ }^{+}$containing one or more ${ }^{13} \mathrm{C}$.

The upper inset in Fig. 1 reveals an abrupt drop in the $\mathrm{C}_{60} \mathrm{He}_{n}{ }^{+}$abundance at $n=32$; the lower inset reveals another one at $n=60$. For a quantitative analysis we fit Lorentzians to all ion peaks; the yield of ${ }^{12} \mathrm{C}_{60} \mathrm{He}_{n}{ }^{+}$is presented in Fig. 2. Statistical uncertainties are smaller than the symbol size. Ion peaks contaminated by impurities have been omitted from the graph [17].

Mass spectra of $\mathrm{C}_{70}$ embedded in helium were recorded and analyzed similarly; the ion yield of ${ }^{12} \mathrm{C}_{70} \mathrm{He}_{n}{ }^{+}$is plotted in Fig. 2. Contamination is more severe, last but not least due to contamination by heavier fullerenes $\left(\mathrm{C}_{76}\right.$, $\mathrm{C}_{78}, \mathrm{C}_{84}$ ). Problematic data points have been omitted [17]. The ion yield drops abruptly at $n=37$ and 62 .

It is tempting to assign the anomalies observed for $\mathrm{C}_{60} \mathrm{He}_{32}{ }^{+}$and $\mathrm{C}_{70} \mathrm{He}_{37}{ }^{+}$to the formation of ordered layers with one helium bound to each of the (12) pentagonal and (20 or 25) hexagonal faces of the fullerene. This geometry would represent the analogue of a hypothetical $1 \times 1$ phase on graphite, with one adatom at the center of each carbon ring. The phase does not form on planar graphite because it would imply a separation of only $2.46 \AA$ between adjacent $\mathrm{He}$ atoms, well below the minimum in their interaction potential at $2.97 \AA$ [18]. The $1 \times 1$ phase becomes accessible for $\mathrm{C}_{60}$ because curvature will increase the distance between adatoms.

An alternative explanation of the observed anomalies might be the completion of a solvation shell. In liquid helium the strong binding to cations leads to the formation of snowballs which greatly reduce the ion mobility [19]. The number of helium atoms in a solvation shell depends on the ionic radius. For atomic cations, $10-20 \mathrm{He}$ fit into the first shell [20]; considerably more should fit around $\mathrm{C}_{60}{ }^{+}$. Perhaps a commensurate layer is completed for

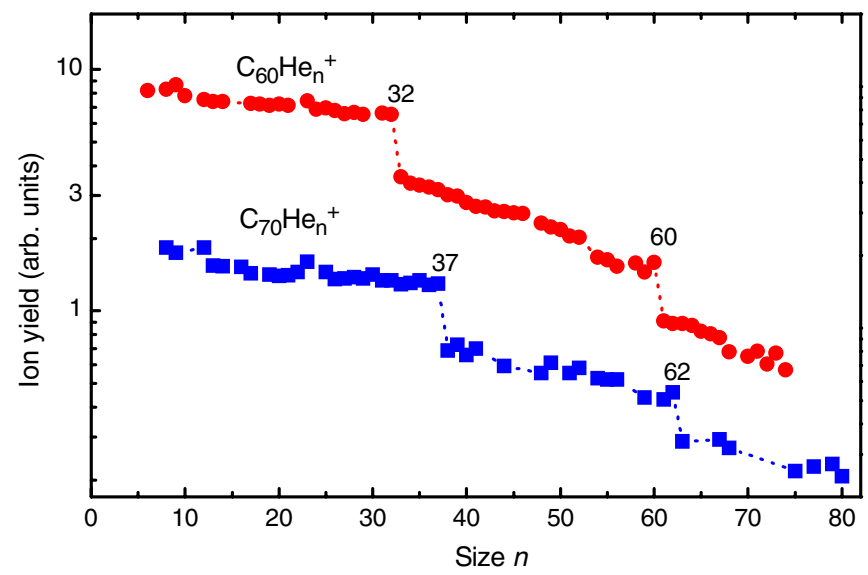

FIG. 2 (color online). Ion yields of ${ }^{12} \mathrm{C}_{60} \mathrm{He}_{n}{ }^{+}$and ${ }^{12} \mathrm{C}_{70} \mathrm{He}_{n}{ }^{+}$ versus size $n$. 
$\mathrm{C}_{60} \mathrm{He}_{32}{ }^{+}$and a solvation shell for $\mathrm{C}_{60} \mathrm{He}_{60}{ }^{+}$, analogous to the formation of the $\sqrt{3} \times \sqrt{3}$ commensurate phase on planar graphite well before completion of the first, disordered monolayer?

We have performed MD simulations of $\mathrm{C}_{60} \mathrm{He}_{n}{ }^{+}$to study the energetics, structure, and dynamics of the adsorbate. In a first step high level ab initio calculations of the helium-benzene complex and the helium dimer are carried out; they serve as benchmarks for the density-functional theory study of the interaction of He with $\mathrm{C}_{60}{ }^{+}$. Becke's 1997 (B97) functional is chosen because it performs best when compared with $a b$ initio calculations. B97/6-31+ $+G(d, p)$ energies of $\mathrm{He}_{-} \mathrm{C}_{60}{ }^{+}$are used as the input database for building the $\mathrm{He}_{-} \mathrm{C}_{60}{ }^{+}$force field; $\mathrm{CCSD}(\mathrm{T}) / \mathrm{CBS}$ (coupled-cluster method with single, double, and perturbative triple excitations, at the complete basis set limit) energies of $\mathrm{He}_{2}$ are used for the $\mathrm{He}-\mathrm{He}$ force field.

The energy of $\mathrm{C}_{60} \mathrm{He}^{+}$(with the $\mathrm{C}_{60}{ }^{+}$geometry being frozen) is calculated for different positions of $\mathrm{He}$; we thus obtain potential energy curves for $\mathrm{He}-\mathrm{C}_{60}{ }^{+}$along various symmetry axes, binding energies at specific positions, and energy barriers between them. The binding energy is largest $(10.3 \mathrm{meV})$ at $3.2 \AA$ above the center of a hexagon ( $H$ position), slightly less $(9.0 \mathrm{meV})$ at $3.2 \AA$ above the center of a pentagon $(P)$, and $5-6 \mathrm{meV}$ when He is located above a vertex $(V)$ or a carbon bond $(H H$ and $H P$ for bonds between hexagon-hexagon and hexagon-pentagon, respectively). Only $H$ and $P$ positions represent local minima; $H H$ positions are highest in energy.

Various starting configurations are tried for the MD simulations, including those that are, based on the $\mathrm{He} \mathrm{C}_{60}{ }^{+}$energies, low- or high-energy configurations. The system is initially heated to $10 \mathrm{~K}$ (a periodic box is applied to prevent escape of $\mathrm{He}$ ), then slowly cooled at a rate of $0.1 \mathrm{~K}$ per $50 \mathrm{ps}$. Once the system reaches $4 \mathrm{~K}$, which is the estimated temperature of $\mathrm{C}_{60} \mathrm{He}_{n}{ }^{+}$under our experimental conditions [21], the simulation is run for another $5 \mathrm{~ns}$ to ensure full equilibration and to collect statistical information.

Figure 3(a) displays the dissociation energies $D_{n}$ of $\mathrm{C}_{60} \mathrm{He}_{n}{ }^{+}$with respect to loss of one He, i.e., the difference between the total energies $E_{n}$ of $\mathrm{C}_{60} \mathrm{He}_{n}{ }^{+}$in their most stable configurations, $D_{n}=-E_{n}+E_{n-1}$. $D_{n}$ remains constant at around $10 \mathrm{meV}$ up to $n=32$ where all hexagon and pentagon sites are filled; it then drops by a factor of 2 , and gradually recovers to $8-9 \mathrm{meV}$ with increasing $n$.

Anomalies in experimental ion yields $I_{n}$ reflect anomalies in dissociation energies $D_{n}$ if ionization is accompanied by strong fragmentation [22]. Experimental values $D_{\text {expt }, n}$ may be deduced from $I_{n}$ if an absolute energy scale can be established and the microcanonical heat capacities are known. However, helium on graphite has a low heat capacity because of a gap in the phonon spectrum [2]; the heat capacity of $\mathrm{C}_{60} \mathrm{He}_{n}{ }^{+}$will be particularly low because at $T=4 \mathrm{~K}$ the $\mathrm{C}_{60}{ }^{+}$substrate will be in its vibrational

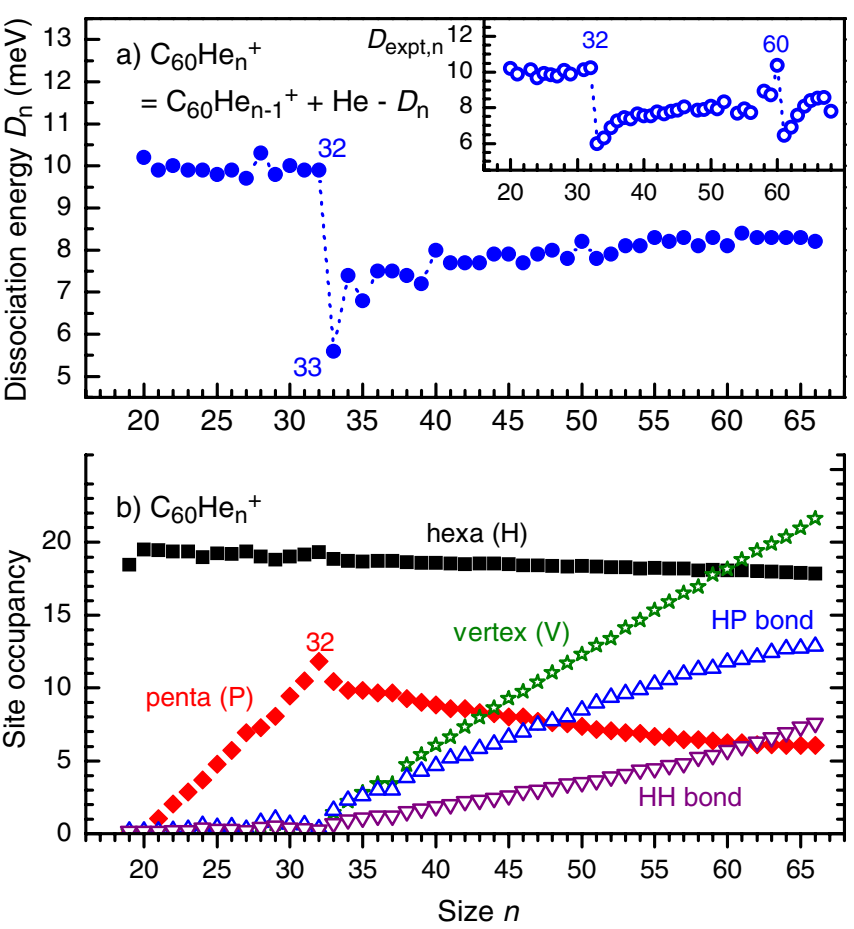

FIG. 3 (color online). (a) Calculated dissociation energies $D_{n}$ for loss of $\mathrm{He}$ from $\mathrm{C}_{60} \mathrm{He}_{n}{ }^{+}$. The inset shows the dissociation energies derived from ion yields. (b) Average site occupancy for $\mathrm{C}_{60} \mathrm{He}_{n}{ }^{+}$.

ground state (the lowest vibrational excitation of $\mathrm{C}_{60}$ is $33 \mathrm{meV}$ ). Assuming zero heat capacities we derive the relation $D_{\text {expt }, n}=I_{n} D_{\text {av }} / I_{\text {av }}$, where $D_{\text {av }}$ and $I_{\text {av }}$ are local averages of $D_{n}$ and $I_{n}$, respectively; they are obtained by weighted averaging over several adjacent sizes [23]. Resulting $D_{\text {expt } n}$ values are plotted in the inset of Fig. 3(a). The close similarity in shape and magnitude of $D_{\text {expt }, n}$ and $D_{n}$ at $n=32$ is encouraging. The anomaly in $D_{\text {expt }, n}$ at $n=60$ which finds no parallel in $D_{n}$ is addressed below.

Structural details emerge from a statistical analysis of the simulation data. Figure 3(b) displays time-averaged occupancies of specific sites. Up to $n=20$ nearly all $\mathrm{He}$ are located in $H$ sites. Beyond $n=20$ excess atoms occupy primarily $P$ sites. Beyond $n=32$ additional atoms are forced into $V, H P$, and $H H$ sites. $V$ positions are favored over $H P$ positions even though the latter are energetically favored in the isolated $\mathrm{He}_{-} \mathrm{C}_{60}{ }^{+}$system. Presumably, the energetics of $V$ and $H P$ positions will change due to crowding once all $H$ and $P$ positions are filled.

The occupancy of $P$ sites shows an intriguing behavior. They reach full occupancy at $n=32$, beyond which their occupancy drops sharply and levels off at about six, i.e., only $50 \%$ of pentagons are occupied whereas the occupancy of hexagons remains high. A different perspective is offered by Fig. 4(a), which shows the number density of helium versus distance $R$ from the $\mathrm{C}_{60}$ center. For $n \leq 32$ all atoms are in registry with the substrate; their radial 

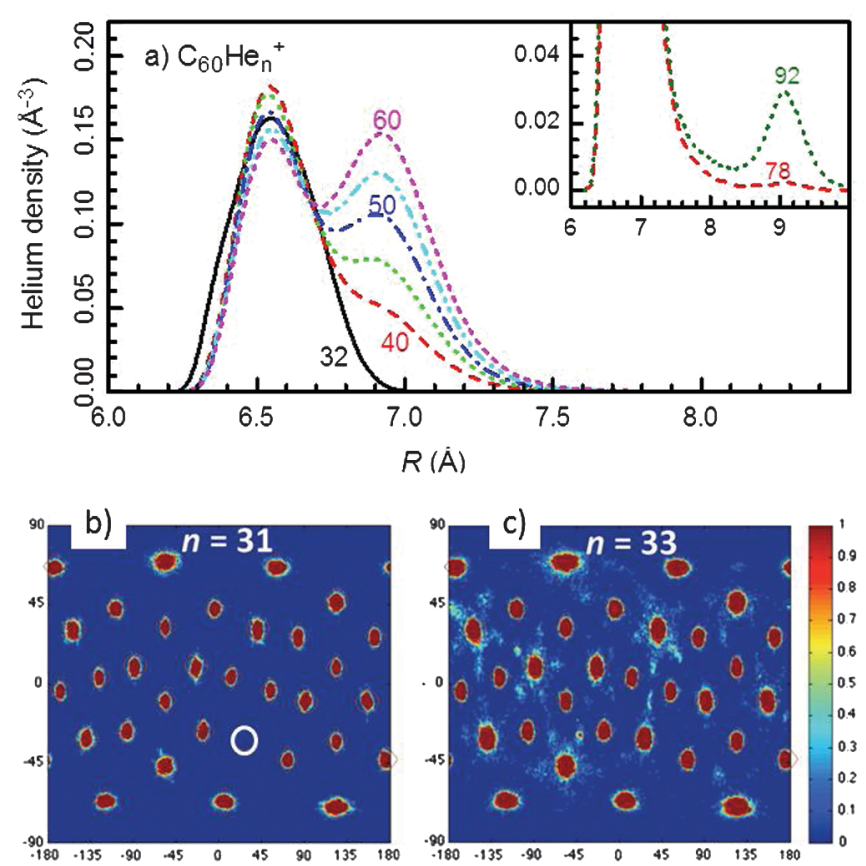

FIG. 4 (color). (a) Number density of helium atoms versus distance $R$ from the fullerene center for $n=$ $32,40,45,50,55,60$. The inset shows the density in the second shell for larger $n$. (b) Angular He distribution for $\mathrm{C}_{60} \mathrm{He}_{31}{ }^{+}$. The white circle marks the unoccupied pentagonal site. (c) Angular distribution for $\mathrm{C}_{60} \mathrm{He}_{33}{ }^{+}$.

distance peaks at $6.5 \AA$. Beyond $n=32$ another peak emerges at $6.9 \AA$ representing atoms that are not in registry.

The small $(6 \%)$ separation between the two peaks in Fig. 4(a) justifies the notion that up to $n=60$ all atoms are confined to the first solvation shell. At what size will atoms occupy a distinct second shell? At $n=32$ the layer is of low density; the average distance between adjacent helium atoms is $4.3 \AA$ for sites over adjacent pentagon and hexagons, and $4.6 \AA$ over adjacent hexagon pairs, even larger than the $4.26 \AA$ between $\mathrm{He}$ atoms in the $\sqrt{3} \times \sqrt{3}$ phase on graphite. Therefore about twice as many atoms $(2 \times 32)$ should fit into the first solvation shell of $\mathrm{C}_{60}{ }^{+}$; the anomaly at $n=60$ in the ion yield and experimental dissociation energy may signal completion of this shell. The anomaly at $n=62$ observed in mass spectra of $\mathrm{C}_{70} \mathrm{He}_{n}{ }^{+}$(see Fig. 2) supports this interpretation: If one estimates the number density of $\mathrm{He}$ in $\mathrm{C}_{70} \mathrm{He}_{62}{ }^{+}$with a continuum approximation, one obtains a value within $1 \%$ of that for $\mathrm{C}_{60} \mathrm{He}_{60}{ }^{+}$.

The MD simulations, though, do not fully confirm this interpretation. The radial distributions [see the inset in Fig. 4(a)] reveal a distinct new peak (at $R=9.0 \AA$ ) at $n=92$. The peak is barely visible at $n=78$ and absent at $n=74$, suggesting that the first solvation shell fills at $n=74$. However, a shortcoming of the MD simulations discussed so far is their neglect of quantum effects. As a remedy, we have generated an effective Feynman-Hibbs potential from our $a b$ initio potential that takes into account quantum effects to second order in $\hbar$ [24]. The dissociation energies obtained for $n \leq 32$ change by less than $10 \%$; the abrupt drop beyond $n=32$ is reduced but still significant. Beyond $n=58$, one can clearly see the formation of a second shell of $\mathrm{He}$ atoms. At $4 \mathrm{~K}$, some of them evaporate after several hundreds of ps, but at $3 \mathrm{~K}$ they stay in the complex. For systems containing the same number of $\mathrm{He}$ atoms in the second shell, the radial distributions are qualitatively the same as those obtained from purely classical MD simulations. Hence the main quantum effect is to reduce the onset for growth of the second shell.

It is worth noting that the second layer is not stable if the effective potential is computed to all orders in $\hbar$. This result is, however, not surprising because it has been shown [25] that, in the framework of Feynman-Hibbs theory, the effective potential calculated to all orders in $\hbar$ significantly overestimates quantum effects. Thus, a further improvement of the present MD simulations would imply the use of full quantum dynamics methods, which is not feasible for systems of this size.

For helium on graphite, commensurate phases are identified by anomalies in the heat capacity versus temperature; the $\sqrt{3} \times \sqrt{3}$ phase exhibits an order-disorder transition at $3 \mathrm{~K}$ [2]. It is not practical to determine the temperature dependence for specific cluster sizes in our (classical) MD simulation, but we can identify qualitative changes versus size at a constant temperature of $4 \mathrm{~K}$. For $n \leq 32$ all $\mathrm{He}$ atoms are nearly immobile. This is demonstrated in Fig. 4(b), which presents the time average of the angular distribution of $\mathrm{He}$ in $\mathrm{C}_{60} \mathrm{He}_{31}{ }^{+}$. The unoccupied pentagonal site is marked by a white circle; the site is not visited during the $5 \mathrm{~ns}$ of the simulation. The angular distribution of $\mathrm{C}_{60} \mathrm{He}_{33}{ }^{+}$[Fig. 4(c)], on the other hand, shows diffuse traces of helium in several regions other than $H$ and $P$ sites. Studies for larger values of $n$ show that atoms at hexagon sites are nearly immobile, atoms at pentagon sites are somewhat mobile (facilitated by the presence of vacancies), while the remaining atoms (at $R \approx 6.9 \AA$ ) are highly mobile. Thus, for $n>32$ at $4 \mathrm{~K}$ one finds coexistence of an ordered, solid phase with a disordered, liquidlike phase.

In conclusion, cationic fullerene-helium systems exhibit phenomena akin to helium on graphite. The positive charge and curvature of the substrate enhance corrugation effects. A commensurate phase of low density forms for $\mathrm{C}_{60} \mathrm{He}_{32}{ }^{+}$ (and $\mathrm{C}_{70} \mathrm{He}_{37}{ }^{+}$) when each carbon polygon is occupied by one helium atom. Additional helium atoms in the first layer partially displace atoms from pentagonal but not hexagonal sites; atoms at hexagonal sites are immobile while other atoms are highly mobile at $4 \mathrm{~K}$. More work is needed to confirm that the anomalies in the experimental data at $\mathrm{C}_{60} \mathrm{He}_{60}{ }^{+}$and $\mathrm{C}_{70} \mathrm{He}_{62}{ }^{+}$are caused by closure of the first solvation shell.

We thank Mare Nostrum BSC and CCC-UAM for computer time. C. L. and P. B. acknowledge a dissertation grant from the vice-rectorate for research of the University of 
Innsbruck; K. H. and H.Z. acknowledge support from the Swedish Research Council. This work was supported by MICINN projects FIS2010-15127, ACI2008-0777, CTQ2010-17006, Consolider-Ingenio CSD2007-00010, CAM program NANOBIOMAGNET S2009/MAT1726, the Austrian Science Fund, Wien (FWF, projects P19073, L633, and I200 N29), the European Commission, Brussels (ITS-LEIF), and the European COST Action CM0702.

Note added in proof.-Recently, we became aware of a theoretical study of melting and freezing of helium clusters doped with $\mathrm{C}_{60}{ }^{+}$[26]. The basic conclusions drawn in this interesting paper closely agree with ours.

*Corresponding author. olof.echt@unh.edu

[1] S. M. Gatica and M. W. Cole, J. Low Temp. Phys. 157, 111 (2009).

[2] D. S. Greywall, Phys. Rev. B 47, 309 (1993).

[3] M. Pierce and E. Manousakis, Phys. Rev. Lett. 81, 156 (1998).

[4] M. C. Gordillo and J. Boronat, Phys. Rev. Lett. 102, 085303 (2009).

[5] S. M. Gatica, M. K. Kostov, and M. W. Cole, Phys. Rev. B 78, 205417 (2008)

[6] J. V. Pearce, M. A. Adams, O. E. Vilches, M. R. Johnson, and H. R. Glyde, Phys. Rev. Lett. 95, 185302 (2005).

[7] A. D. Lueking and M. W. Cole, Phys. Rev. B 75, 195425 (2007); S. M. Gatica, A. D. Lueking, M. W. Cole, and G. D. Mahan, Phys. Rev. B 76, 085406 (2007).

[8] M. C. Gordillo, Phys. Rev. Lett. 101, 046102 (2008).

[9] S. O. Diallo, B. Fak, M. A. Adams, O.E. Vilches, M. R. Johnson, H. Schober, and H. R. Glyde, Europhys. Lett. 88, 56005 (2009).

[10] Z. H. Wang, J. Wei, P. Morse, J. G. Dash, O. E. Vilches, and D. H. Cobden, Science 327, 552 (2010).

[11] B. R. Gibbons, M. Z. Xu, and Z. Bacic, J. Phys. Chem. A 113, 3789 (2009); H. D. Whitley, J. L. DuBois, and K. B. Whaley, J. Phys. Chem. A 115, 7220 (2011).
[12] E. S. Hernandez, M. W. Cole, and M. Boninsegni, Phys. Rev. B 68, 125418 (2003); M. Barranco, E. S. Hernandez, R. Mayol, and M. Pi, Phys. Rev. B 69, 134502 (2004).

[13] Y. Kwon and H. Shin, Phys. Rev. B 82, 172506 (2010).

[14] Y. Kwon, D. M. Ceperley, and K. B. Whaley, J. Chem. Phys. 104, 2341 (1996).

[15] J.P. Toennies and A. F. Vilesov, Angew. Chem., Int. Ed. Engl. 43, 2622 (2004).

[16] C. Leidlmair, P. Bartl, H. Schöbel, S. Denifl, M. Probst, P. Scheier, and O. Echt, Astrophys. J. Lett. 738, L4 (2011).

[17] ${ }^{12} \mathrm{C}_{60} \mathrm{He}_{n}{ }^{+}$was considered contaminated based on the peak width, intensity relative to ${ }^{13} \mathrm{C}^{12} \mathrm{C}_{59} \mathrm{He}_{n}{ }^{+}$, or the presence of a strong peak at $1 \mathrm{u}$ lower mass.

[18] Data complied by K. P. Huber and G. Herzberg, NIST Chemistry Webbook, http://webbook.nist.gov/cgi/cbook .cgi? $\mathrm{ID}=\mathrm{C} 12184984 \&$ Units=SI \&Mask=1000\#Diatomic (retrieved November 17, 2011).

[19] K. R. Atkins, Phys. Rev. 116, 1339 (1959); P. Moroshkin, V. Lebedev, and A. Weis, Phys. Rev. Lett. 102, 115301 (2009); E. Coccia, E. Bodo, and F. A. Gianturco, Europhys. Lett. 82, 23001 (2008); P. Slavicek and M. Lewerenz, Phys. Chem. Chem. Phys. 12, 1152 (2010).

[20] F. Ferreira da Silva, P. Waldburger, S. Jaksch, A. Mauracher, S. Denifl, O. Echt, T.D. Märk, and P. Scheier, Chem. Eur. J. 15, 7101 (2009); L. An der Lan, P. Bartl, C. Leidlmair, R. Jochum, S. Denifl, O. Echt, and P. Scheier, Chem. Eur. J. (to be published).

[21] The temperature of clusters in an evaporative ensemble is proportional to their binding energy [C. E. Klots, Nature (London) 327, 222 (1987)]. Helium droplets cool to $0.37 \mathrm{~K}$ [15]; their binding energy $(0.62 \mathrm{meV})$ is an order of magnitude weaker than that of $\mathrm{C}_{60} \mathrm{He}_{n}{ }^{+}$.

[22] C. E. Klots, Z. Phys. D 21, 335 (1991); K. Hansen and U. Näher, Phys. Rev. A 60, 1240 (1999).

[23] S. Prasalovich, K. Hansen, M. Kjellberg, V. N. Popok, and E. E. B. Campbell, J. Chem. Phys. 123, 084317 (2005).

[24] N. Tchouar, F. Ould-Kaddour, and D. Levesque, J. Chem. Phys. 121, 7326 (2004).

[25] L. M. Sese, Mol. Phys. 81, 1297 (1994).

[26] F. Calvo, Phys. Rev. B 85, 060502(R) (2012). 